\title{
A Rotating Metal Band Target for Pion Production at Neutrino Factories and Muon Colliders*
}

\author{
B.J. King ${ }^{\dagger}$, N. Simos, R.V. Weggel, BNL, Upton, NY 11973-5000, USA \\ N.V. Mokhov, FNAL, Batavia, IL 60510-0500, USA
}

\begin{abstract}
A conceptual design is presented for a high power pion production target for neutrino factories and muon colliders that is based on a rotating metal band of either inconel alloy 718 , titanium alloy $6 \mathrm{Al}-4 \mathrm{~V}$ grade 5 or nickel. The band is 5 meters in diameter and is tangentially intercepted by a pulsed proton beam inside a 20 Tesla tapered solenoidal magnetic pion capture channel. The mechanical layout and cooling setup of the target are summarized and results are presented from realistic MARS and ANSYS computer simulations of pion yields, energy depositions and shock heating stresses. The target scenario is predicted to perform satisfactorily and with conservative safety margins for multimegawatt pulsed proton beams.
\end{abstract}

\section{INTRODUCTION}

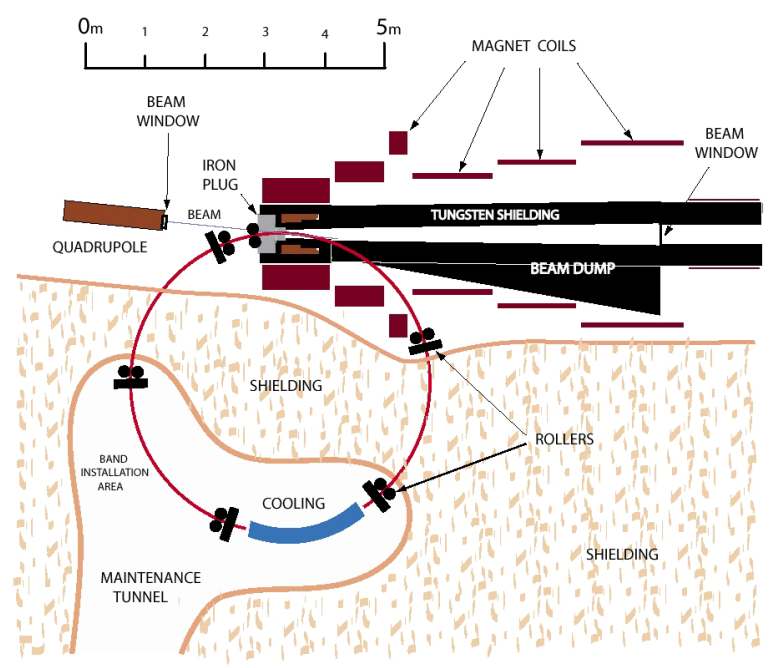

Figure 1: A conceptual illustration of the targetry setup.

This paper presents a target option for pion production at neutrino factories and muon colliders that is based around a rotating band geometry $[1,2,3,4,5,6]$. It summarizes the material in reference [6].

\section{MECHANICAL DESIGN SCENARIO}

A plan view of the band target design scenario is shown in Fig. 1. A 2.5 meter radius circular target band threads

\footnotetext{
* This work was performed under the auspices of the U.S. Departmen of Energy under contract no. DE-AC02-98CH10886.

†email: bking@bnl.gov .
}

through a solenoidal magnetic capture channel $[7,8,4]$ to tangentially intercept the proton beam.

Three metals with track records as production targets or beam windows are considered as candidates for the target band: inconel 718 (a niobium-modified nickel-chromiumiron "superalloy"), titanium alloy $6 \mathrm{Al}-4 \mathrm{~V}$ grade 5 and pure nickel.

The cross sectional dimensions of the band and its orientation relative to the proton beam for each of the 3 candidate materials are shown in Fig. 2, and the proton beam dimensions and the specifications and material properties of the band are enumerated in Table 1.

The band is in an air environment and is guided and driven by several sets of rollers at rotation speeds of order $1 \mathrm{~m} / \mathrm{s}$. Faster rotation minimizes heating pile-up from successive proton pulses but increases the eddy current drag in the 20 Tesla solenoid. A procedure has been developed $[5,6]$ for its routine installation and extraction.

Typically, approximately $7 \%$ of the proton beam energy is deposited in the band target as heat. The heated portion of the band rotates through a 2 meter long water cooling tank, as shown in Fig. 3. The flow rate and heat transfer parameters are relatively comfortable [6] even for few-MW proton beams - a flow rate of a few liters per second and heat transfer of some tens of $\mathrm{W} / \mathrm{cm}^{2}-$ so forced convection is not required.

\section{SIMULATIONS OF YIELD, ENERGY DEPOSITION AND STRESS}

Probably the most critical issue faced in solid-target design scenarios for pion production at neutrino factories or muon colliders is the survivability and long-term structural integrity of solid targets in the face of repeated shock heating. A critical design parameter for assessing the shock stresses on the band is the energy contained in each bunch of the pulsed proton beam. The bunch repetition rate is less important because the beam-induced shock waves die down quickly enough for the bunches to be relatively independent in any reasonable muon collider bunch scenario.

Full MARS [11] tracking and showering Monte Carlo simulations of pion yields and energy deposition densities were conducted for incident proton bunches at representative $6 \mathrm{GeV}$ and $24 \mathrm{GeV}$ beam energies. Three-dimensional energy deposition maps from the MARS simulations were then used as input to finite element stress simulations using the commercial code ANSYS.

Fig. 4 shows the ANSYS predictions for von Mises stress development in the bands and Table 1 summarizes 

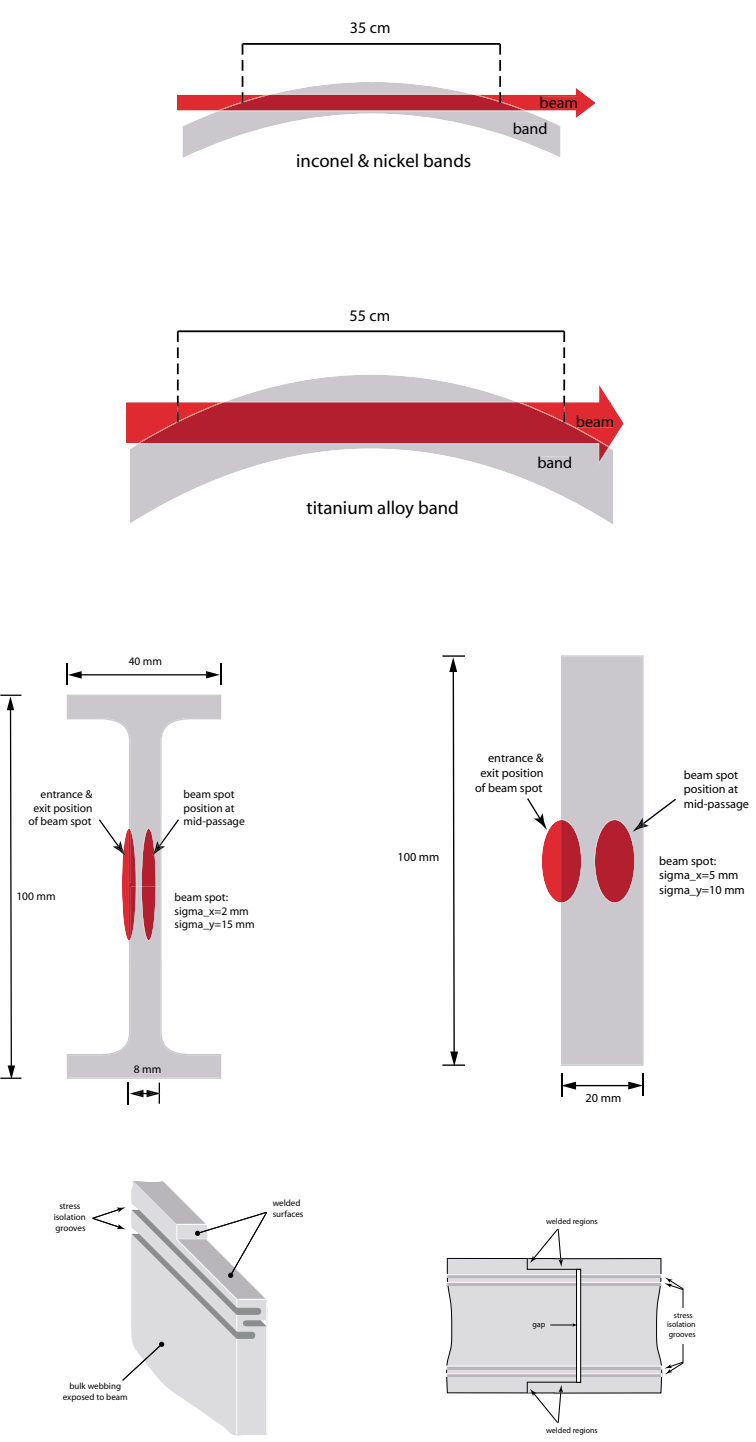

Figure 2: Several views of the target bands and position of the proton beam: plan views for the inconel or nickel bands (top) and for titanium (2nd row), with a vertical:horizontal aspect ratio of approximately 5.4:1; cross-sectional views for inconel or nickel bands (left, 3rd row) and for the titanium alloy band option (right, 3rd row); and threedimensional (bottom left) and side (bottom right) views showing how the welds between band segments are mechanically isolated from beam-induced shock waves.

the yield, energy deposition and stress predictions from the MARS and ANSYS simulations. These have been benchmarked to muon colliders with the largest proton bunch charge normally considered [7] at collision: $4 \times 10^{12}$ muons per bunch, which implies $[7,6]$ initially capturing a total of approximately $3.2 \times 10^{13}$ pions and muons. where both signs have been summed. The beam-induced shock stresses at neutrino factories are typically less demanding $[4,5]$.

It is seen from the predicted stresses as fractions of the fatigue strength that the viability of inconel bands becomes

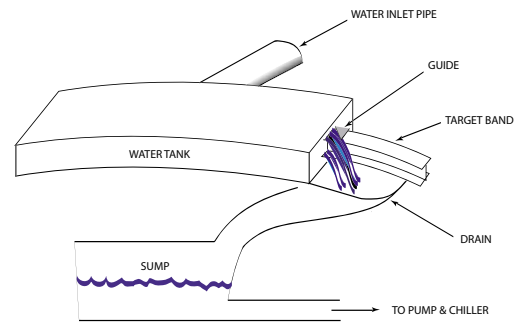

Figure 3: A conceptual illustration of the target cooling setup.

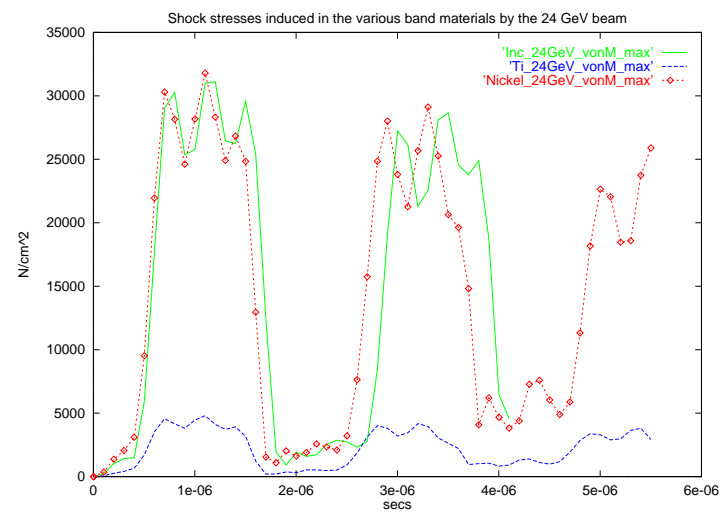

Figure 4: Predicted time dependence of maximum von Mises stresses on inconel 718, titanium alloy and nickel bands due an instantaneous energy deposition from a bunch of $1.5 \times 10^{14} 6 \mathrm{GeV}$ protons with transverse dimensions as given in Table 1 .

borderline (53-69\%) for the most stringent muon collider production assumptions but the titanium alloy should still provide a very conservative safety margin (10-14\%), albeit with a pion yield about $20 \%$ lower than the other two candidate materials. Since successfully operating nickel targets [12] seem to evade predictions for low yield strengths, the suitability of nickel bands is not well predicted by these simulations and would need to instead be determined in test beam experiments.

\section{CONCLUSIONS}

In summary, the rotating metal band target concept appears to be a promising option for pion production targets at neutrino factories and muon colliders. The engineering aspects look manageable and initial simulations of pion yields and target stresses are encouraging for each of three candidate target band materials: inconel 718 , titanium alloy $6 \mathrm{Al}-4 \mathrm{~V}$ grade 5 and nickel.

\section{REFERENCES}

[1] A Cupronickel Rotating Band Pion Production Target for Muon Colliders, B.J. King et al., Proc. PAC'99, IEEE, pp. 3041-3.

[2] Rotating Band Pion Production Targets for Muon Colliders and Neutrino Factories, B.J. King, NIM A 451 
Table 1: Specifications of the target band and assumed proton beam parameters, a tabulation of some relevant properties of the candidate band materials, and a summary of MARS and ANSYS predictions for pion yields, energy depositions and stresses. Units are indicated in square brackets. See text for definitions and details.

\begin{tabular}{|c|c|c|c|c|c|c|}
\hline Property & \multicolumn{2}{|c|}{ inconel 718} & \multicolumn{2}{|c|}{ Ti-alloy } & \multicolumn{2}{|c|}{ nickel } \\
\hline target band radius, $[\mathrm{m}]$ & \multicolumn{2}{|c|}{2.5} & \multicolumn{2}{|c|}{2.5} & \multicolumn{2}{|c|}{2.5} \\
\hline band thickness, [mm] & \multicolumn{2}{|c|}{8} & \multicolumn{2}{|c|}{20} & \multicolumn{2}{|c|}{8} \\
\hline band webbing height, [mm] & \multicolumn{2}{|c|}{100} & \multicolumn{2}{|c|}{100} & \multicolumn{2}{|c|}{100} \\
\hline full width of band flanges, [mm] & \multicolumn{2}{|c|}{40} & \multicolumn{2}{|c|}{-} & \multicolumn{2}{|c|}{40} \\
\hline beam path length in band, [cm] & \multicolumn{2}{|c|}{35} & \multicolumn{2}{|c|}{55} & \multicolumn{2}{|c|}{35} \\
\hline proton interaction lengths $(\lambda)$ & \multicolumn{2}{|c|}{2.1} & \multicolumn{2}{|c|}{2.0} & \multicolumn{2}{|c|}{2.3} \\
\hline weight of band, [kg] & \multicolumn{2}{|c|}{169} & \multicolumn{2}{|c|}{139} & \multicolumn{2}{|c|}{183} \\
\hline horizontal beam-channel angle $(\alpha)$, [mrad] & \multicolumn{2}{|c|}{100} & \multicolumn{2}{|c|}{100} & \multicolumn{2}{|c|}{100} \\
\hline rms beam spot size at target (horizontal), $[\mathrm{mm}]$ & \multirow{2}{*}{\multicolumn{2}{|c|}{$\begin{array}{c}2 \\
15\end{array}$}} & \multirow{2}{*}{\multicolumn{2}{|c|}{$\begin{array}{c}5 \\
0\end{array}$}} & & \\
\hline rms beam spot size at target (vertical), $[\mathrm{mm}]$ & & & & & & \\
\hline average atomic number, $\mathrm{Z}$ & & & & & & \\
\hline average atomic weight, $\mathrm{A}$ & & & & & & \\
\hline density $(\rho),\left[\mathrm{g} \cdot \mathrm{cm}^{-3}\right]$ & & & & & & \\
\hline interaction length $(\lambda),[\mathrm{cm}]$ & & & & & & \\
\hline radiation length $\left(\mathrm{X}_{0}\right),[\mathrm{cm}]$ & & & & & & \\
\hline melting point, $\left[{ }^{\circ} \mathrm{C}\right]$ & & & & & & \\
\hline heat capacity, $\left[J . K^{-1} \cdot \mathrm{g}^{-1}\right]$ & & & & & & \\
\hline thermal conductivity, $\left[\mathrm{W} \cdot \mathrm{m}^{-1} \mathrm{~K}^{-1}\right.$ ] & & & & & & \\
\hline electrical conductivity, $\left[\mathrm{MS} \cdot \mathrm{m}^{-1}\right]$ & & & & & & \\
\hline thermal expansion coefficient $(\alpha),\left[10^{-5} / \mathrm{K}\right]$ & & & & & & \\
\hline elastic modulus $(\mathrm{E}),\left[10^{11} \mathrm{~N} / \mathrm{m}^{2}\right]$ & & & & & & \\
\hline $0.2 \%$ yield strength, $[\mathrm{MPa}]$ & 110 & [10] & $\sim 9$ & $0,13]$ & & 10] \\
\hline fatigue strength $[\mathrm{MPa}]$, no. cycles & $480-620$ & $10^{8}[9]$ & $510-700$ & ${ }^{7}[10,13]$ & & \\
\hline proton energy [GeV] & 6 & 24 & 6 & 24 & 6 & 24 \\
\hline captured $\pi^{+}$yield/proton & 0.102 & 0.303 & 0.080 & 0.249 & 0.102 & 0.105 \\
\hline captured $\pi^{-}$yield/proton & 0.105 & 0.273 & 0.083 & 0.224 & 0.302 & 0.292 \\
\hline protons per pulse, $\mathrm{ppp}^{3.2}\left[10^{13}\right]$ & 15.5 & 5.56 & 19.6 & 6.78 & 15.5 & 5.39 \\
\hline proton pulse energy, $E_{\text {pulse }}^{3.2}[\mathrm{~kJ}]$ & 149 & 214 & 188 & 260 & 149 & 207 \\
\hline max. energy density, $U_{\max }^{3.2}[\mathrm{~J} / \mathrm{g}]$ & 32.0 & 31.7 & 25.6 & 21.3 & 32.5 & 37.4 \\
\hline $\max$. temp. rise, $\Delta T_{\max }^{3.2}\left[{ }^{\circ} \mathrm{C}\right]$ & 74 & 73 & 49 & 40 & 71 & 81 \\
\hline max. von Mises stress, $V M_{m a x}^{3.2}[\mathrm{MPa}]$ & 330 & 360 & 72 & 68 & 330 & 340 \\
\hline$\%$ of fatigue strength & $53-69 \%$ & $58-75 \%$ & $10-14 \%$ & $10-13 \%$ & N.A. & N.A. \\
\hline
\end{tabular}

(2000) pp. 335-343, Proc. ICFA/ECFA Workshop "Neutrino Factories based on Muon Storage Rings (nuFACT'99)", physics/0005007.

[3] Some thoughts on a high-power, radiation cooled, rotating toroidal target for neutrino production, J.R.J. Bennett, NIM A 451 (2000) pp. 344-348, Proc. ICFA/ECFA Workshop "Neutrino Factories based on Muon Storage Rings (nuFACT'99)".

[4] Report from the Neutrino Factory and Muon Collider Collaboration Feasibility study II, http://www.cap.bnl.gob/mumu/studyii .

[5] A Rotating Band Target for Pion Production at a Neutrino Factory, using Study II Parameters, B.J. King, N.V. Mokhov, N. Simos, R.J. Weggel, Muon Collider Note 199, http:www-mucool.fnal.gov/notes/notes.html .

[6] A Rotating Band Target for Pion Production at Muon Colliders, B.J. King, N.V. Mokhov, N. Simos and R.V. Weggel, Proc. 6-Month Feasibility Study on High Energy Muon Colliders; Oct'00-Apr'01, publication on CDs by Rinton Press.
[7] The Muon Collider Collaboration, Status of Muon Collider Research and Development and Future Plans, Phys. Rev. ST Accel. Beams 2, 081001, 3 August, 1999.

[8] FNAL Feasibility Study on a Neutrino Source Based on a Muon Storage Ring,

http://www.fnal.gov/projects/muon_collider/nu-factory/ .

[9] Huntington Alloys - Inconel Alloy 718, 1978.

[10] The elemental composition and other properties of inconel 718 can be found at http://www.matweb.com .

[11] N.V. Mokhov, "The MARS Code System User's Guide", Fermilab-FN-628 (1995). N.V. Mokhov and O.E. Krivosheev, "MARS Code Status", Fermilab-Conf-00/181 (2000). http://www-ap.fnal.gov/MARS/ .

[12] S. O'Day et al., "New Target Results from the FNAL Antiproton Source", Proc. IEEE 1993 PAC 4, 3096.

[13] Trans World Alloys, http://www.twalloys.com . 